\title{
Graduate teaching assistants' views of broken-into-parts physics problems: Preference for guidance overshadows development of self-reliance in problem solving
}

\author{
Melanie Good®, ${ }^{1}$ Emily Marshman $\odot,{ }^{1}$ Edit Yerushalmi $\odot,{ }^{2}$ and Chandralekha Singh ${ }^{1}$ \\ ${ }^{1}$ Department of Physics and Astronomy, University of Pittsburgh, Pittsburgh, Pennsylvania 15260, USA \\ ${ }^{2}$ Department of Science Teaching, Weizmann Institute of Science, \\ 234 Herzl Street, Rehovot, Israel 7610001
}

(Received 2 September 2019; accepted 20 April 2020; published 22 May 2020)

\begin{abstract}
We examined physics graduate teaching assistants' views about introductory physics problem "types," i.e., different ways of posing the same underlying physics problem, within the context of a semester-long teaching assistant (TA) professional development course. Here, we focus on TAs' views about two types of broken-into-parts problems that involve the same underlying physics scenario. One of these problem types does not involve explicit calculation, while the other does. The TAs were asked to list the pros and cons of these two types of broken-into-parts problems, rank them compared to other problem types (e.g., traditional textbook problem not broken-into-parts, context-rich problem, and multiple-choice problem) with the same underlying scenario in terms of their instructional benefit and the level of challenge they might produce for their students, and describe when and how likely they would be to use these types of problems in their own classes in different instructional situations if they had complete control of teaching the class. TAs reported that they found the broken-into-parts problem type to be the most instructionally beneficial out of all the problem types because of the guidance such problems offer, and would use a broken-into-parts problem type often and in a variety of ways (e.g., homework assignments, exams, and quizzes). While providing guidance to students is an appropriate instructional approach, our findings from interviews suggest that many TAs may be motivated to assign broken-into-parts problems out of a desire to make the problem-solving process easy and/or less stressful for students, especially because they felt that introductory students may not be capable of breaking a problem into subproblems on their own. The instructional benefits of gradually removing the scaffolding support to help students develop self-reliance in solving problems appeared to be overlooked by most TAs. In particular, in written responses or in interviews, most TAs did not mention a long-term goal of helping students develop more independence in problem solving for which one may start with broken-into-parts problems and gradually transition to problems that are not broken into parts. While the study findings that provide a snapshot of TA views in the middle and at the end of a TA professional development course may only apply to graduate TAs at a similar large university, at those institutions, professional development of TAs should take into account these findings and help TAs reflect on the important role that removing scaffolding support gradually and providing adequate challenge can play in helping introductory students develop self-reliance and become independent, expertlike problem solvers.
\end{abstract}

DOI: 10.1103/PhysRevPhysEducRes.16.010128

\section{INTRODUCTION}

The desired learning goals for students in many introductory physics courses often include learning physics concepts and developing expertise in problemsolving and reasoning skills, see e.g., Refs. [1-17]. The cognitive apprenticeship model can serve as a useful model to support these goals. In this field-tested

Published by the American Physical Society under the terms of the Creative Commons Attribution 4.0 International license. Further distribution of this work must maintain attribution to the author(s) and the published article's title, journal citation, and DOI. framework, learning takes place through a guided process in which students gradually develop self-reliance in solving problems on their own. To facilitate this process, the cognitive apprenticeship model includes three major components: modeling by an instructor or expert to demonstrate the criteria of good performance in problem solving, coaching, and scaffolding to provide immediate feedback as students engage in problem solving, and weaning the support to build autonomous expertlike problem-solving ability [18].

\section{A. Expert vs novice problem solving}

Physics experts, e.g., physics faculty members, organize their physics knowledge hierarchically so that 
underlying concepts are connected in a meaningful and structured way [1-16] and they exhibit positive approaches and attitudes towards scientific problem solving. An expertlike, efficient problem-solving approach involves systematically analyzing a problem and planning a solution path (including drawing a diagram, decomposing the problem into subproblems, and carefully contemplating the knowns and unknowns), implementing the solution plan, and checking the results [19-24]. Experts employ a systematic problem-solving process and their underlying knowledge structure that is well connected and organized in a hierarchical manner aids this process [25]. The use of effective problem solving heuristics and organization of knowledge hierarchically in schema allow experts to reduce their cognitive load during the problemsolving process and solve problems more effectively and efficiently [26].

While physics experts' knowledge, including how the knowledge is structured in well-organized schema, and their effective approaches to problem solving facilitates the problem-solving process, many introductory physics students use novicelike approaches while solving physics problems [25]. Also, many introductory physics students view physics as a collection of disconnected facts and equations [25]. Without explicit guidance, they often employ a problem-solving process which is not systematic and display an underlying knowledge structure that is fragmented and not well organized [25]. They often resort to immediately searching for an equation or formula that may be relevant to the problem at hand and putting given values into the formula in the hopes of obtaining a correct final answer without doing deeper contemplation and conceptual analysis and planning of the solution. In particular, without scaffolding support, the deliberate act of performing a careful conceptual analysis and decomposing a physics problem into more manageable subproblems that can facilitate the problem-solving process does not often come naturally for novice problem solvers.

To develop expertise in problem solving, students can benefit from being explicitly instructed on how to use effective problem-solving strategies while solving physics problems and learning physics [19-24]. Studies have shown that when students are deliberately taught to follow a systematic problem-solving approach, they outperform students who are not taught to solve problems in a systematic manner on challenging follow-up problems [19-24]. For example, in one study, students were asked to first construct a problem solution plan and only then they implemented the plan and reflected upon the problemsolving process [23]. When students were taught such a systematic approach, they performed significantly better on increasingly challenging problems than students with similar prior knowledge who were not taught to follow a systematic approach [23].

\section{B. The role of different problem types in the development of expertise via the cognitive apprenticeship model}

It is clear from prior research that in order to help students develop expertlike problem-solving approaches, it is beneficial to give them explicit guidance and scaffolding support [19-23]. This systematic problem-solving approach that students must be taught explicitly using the cognitive apprenticeship model should begin with helping them in carefully analyzing the problem conceptually and planning the solution (e.g., by drawing a diagram and transforming the problem into a representation that makes further analysis easier, thinking about the knowns and unknowns and dividing the problem into subproblems, contemplating the laws of physics that may be useful for each subproblem and why that is the case, predicting some of the features of the solution that can be checked after solving the problem) before embarking on the implementation of the plan. Moreover, students should also be explicitly guided to learn that after implementing the plan, the problem solver should do a reasonability check to evaluate if the solution makes sense and reflect upon why certain laws of physics were useful in that context while others were not, and how they would recognize that the same law of physics should be used in a future physics problem that may have a different context or surface feature.

All major components of the cognitive apprenticeship model (i.e., modeling, coaching and scaffolding, and weaning) are crucial to the development of expertise in problem solving $[18,27,28]$. Thus, with the goal of helping students become experts, different problem types, i.e., different ways in which a physics problem is posed, can facilitate various aspects of the cognitive apprenticeship model and help students develop expertlike problemsolving skills and learn physics. In particular, the choices that instructors make about the types of problems to use with their students may allow for different aspects of the guided process to unfold and can also facilitate different aspects of learning a systematic problem-solving approach. For example, students need opportunities to see a systematic approach modeled for them so that they can develop an understanding of what is required in solving problems in an effective way (criteria of good performance). They also need to receive coaching and scaffolding support through the process of systematic problem solving at different stages of expertise development so that they can practice problem solving while receiving immediate feedback on how to improve.

Problems that provide built-in support and/or modeling, e.g., broken-into-parts problems, may be beneficial for the modeling and coaching aspects of student learning. However, after modeling and coaching and scaffolding, students also need opportunities to experience removal of the support so that they can be weaned into more independent execution of a systematic problem-solving approach. 
For the weaning aspect, when self-reliance is being developed, problems that provide less in the way of built-in support can be useful.

The reason a physics problem that is broken into parts may be useful in the initial modeling and coaching of the expertlike problem-solving approaches in a particular context is that these types of problems are less overwhelming for novices during the initial modeling and coaching phase. In particular, when initially modeling and coaching students to learn to conduct conceptual analysis of the problems and plan the solutions, brokeninto-parts problems are more manageable than the same underlying problems which are not broken into parts because instructors or TAs can demonstrate and coach students through the steps one should take in conceptually analyzing the problems and formulating logical problem solutions. Specifically, broken-into-parts problems can be used as a model for students to learn how to decompose a problem into smaller subproblems and provide them support in managing a complex problem. In addition, broken-into-parts problems afford the opportunity for instructors to provide coaching, scaffolding, and immediate support since such problems allow for student difficulties at each step to be readily identified. Then, instructors can provide targeted feedback to students. Thus, broken-intoparts physics problems can be used to help students adopt a systematic problem-solving approach by modeling the process and coaching them regarding how to proceed in breaking a problem into subproblems. In other words, broken-into-parts problems in a particular context can be a great tool to scaffold student learning and help them develop expertlike problem-solving skills.

Nevertheless, to ensure development of expertlike problem-solving skills and help students become good problem solvers, the weaning stage of the cognitive apprenticeship model is also important [18]. In particular, while brokeninto-parts problems can serve as a model for learning to decompose physics problems into subproblems and can provide opportunities to coach students, they do not readily offer the opportunity for students to engage in the conceptual analysis and decomposition process of a problem into subproblems independently. Thus, this type of problem is not effective for the weaning aspect of the cognitive apprenticeship model, an aspect that is crucial for helping students develop self-reliance in solving complex physics problems. If students are mostly given problems which are broken into parts in homework, quizzes, and exams, they will not have many opportunities or incentive to practice decomposing problems into subproblems on their own to gain problem-solving independence. Therefore, for the weaning phase and helping students develop self-reliance in complex problem solving, other problem types for the same physics scenario can be more beneficial [21].

A problem type that provides less support, e.g., one that is not broken into parts, can help with the weaning aspect of the cognitive apprenticeship model if used after modeling and coaching with broken-into-parts problems. These types of problems can provide opportunities for students to develop a greater level of confidence and self-reliance in expertlike problem solving. We note that weaning is a gradual process and initially when students struggle with problems that are not broken into parts, they will need to be provided appropriate guidance and scaffolding support in order to be able to break the problems into subproblems on their own. Similarly, context-rich problems $[21,22]$ are not generally broken into parts and can vary in difficulty significantly depending on how much redundant (not useful for solving the problems) information is provided, how many subproblems there are in the problems, and whether what students have to solve for is explicitly mentioned or they have to figure it out on their own. These types of context-rich problems could be very useful initially for physics expertise development when students are working in small groups. However, even if students have developed facility with broken-into-parts problems in a particular context, they will need to be provided scaffolding support and coached in order to develop facility with problems not broken into parts before being completely weaned off the support.

\section{The role of teaching assistants in promoting and supporting student learning, and the theoretical framework for our research}

Physics graduate teaching assistants are often employed, especially at large research universities. Their tasks usually involve helping with grading, instructing recitations, and implementing and/or designing quizzes, examples, and other materials for introductory physics students. Furthermore, it has been noted that even though TAs are often responsible for a significant portion of undergraduate instruction, their training for this role is often limited $[29,30]$. In particular, despite the widespread use of TAs and their role in teaching students, limited training is typically provided for TAs and they often carry out their responsibilities without much guidance or support [31]. Only a small minority of physics departments in the U.S. provide semester-long TA professional development, and the majority of physics departments provide only very short training (i.e., a few hours) to prepare the TAs for these various teaching responsibilities [31]. Prior research also suggests that the teaching practices used by TAs are often affected by their workload and their prior beliefs about learning and teaching [32-37]. Additionally, TAs are potential future faculty members whose teaching roles could expand in time. For these reasons, it is important to investigate TAs' views in order to inform their professional development activities related to teaching and learning.

Some TAs may be responsible for choosing the types of problems to use with introductory students, e.g., in 
designing quizzes for their students to take during recitation or discussion or creating example problems to discuss. Moreover, as potential future faculty, TAs may have an ongoing decision-making responsibility about the types of problems to use with their future students [32,33,38-46]. Thus, with limited opportunities for professional development and training in the intervening time between the TA role and the faculty role, TAs' perceptions may also shed light on their perceptions as future faculty members.

The theoretical framework that inspired this research is that it is important to first investigate TAs' perceived ideas about various aspects of teaching in order to provide them suitable professional development opportunities to reflect critically upon the purposes and goals of instruction and the importance, e.g., of evidence-based approaches in bridging the gap between teaching and learning in different instructional situations to meet the goals. Since TAs are not blank slates, their views about teaching including the manner in which they themselves have been instructed in the past can strongly influence their views about different aspects of teaching and learning. Many physics instructors teach primarily using traditional lectures and a significant portion of the recitation time is spent with the TAs solving different example problems on the board for introductory physics students. Therefore, such experiences in their own undergraduate years can greatly shape TAs' views about how students should be taught in different instructional settings. Other prior experiences and concerns about students may also shape TAs' views about employing various instructional approaches, e.g., their views about the perceived instructional affordances and constraints of different types of problems pertaining to a given physics scenario to meet different instructional goals in a variety of situations.

Because different physics problem types, which involve posing the same physics scenario in different ways, can support different aspects of the cognitive apprenticeship model, the use of different problem types in physics courses can impact the effectiveness of instruction and, ultimately, student learning. Making choices about the use of different problem types in various instructional situations to meet different goals often is one of the responsibilities of both faculty and graduate teaching assistants. Therefore, these choices may depend upon the perceived instructional affordances and constraints of posing a problem in a certain manner. Thus, it is important to understand the views of those responsible for making decisions about which physics problem types to use in their introductory physics courses in different instructional situations to meet different goals. The perceived pros and cons of posing an introductory physics problem in different ways and in different instructional contexts can inform activities designed to improve professional development efforts and to help ensure reflection on and recognition of the pedagogical value of posing the same problem in various ways.

\section{Physics faculty members views about broken-into-parts problems}

A prior study was conducted about physics instructors' views regarding different problem types in which they were presented with the same problem types (including the broken-into-parts problems) given to the TAs in the current study [47]. During this prior study, instructors were interviewed using a structured interview protocol in which they were asked to make judgements about the different types of problems [47]. It was found that the instructors generally valued different problem types intended to develop different aspects of expertlike problem solving but their reported use of different problem types in their classes did not always reflect their beliefs regarding the instructional benefits of various problem types. Instructors had differing opinions about the merits of the broken-intoparts problem type. More than half of the instructors felt that it was important to lead students through a problem by breaking it up into subproblems for the students, while slightly less than half of the instructors felt that students benefit from not providing such a guide. Nevertheless, the majority of instructors reported widely using the brokeninto-parts problem type in homework, quizzes, and exams, even if they had reservations about such problems, stating that using such problems would help avoid stressful situations for students [47].

\section{E. Prior work on TAs' professional development and views about teaching and learning}

Several studies have investigated TAs' views about teaching and learning [48-52]. Prior research suggests that there are discrepancies between physics graduate TAs' perceptions of the kinds of teaching strategies that are beneficial for student learning and many of the findings of physics education research. For example, TAs have been found to struggle with the idea that effective grading practices that encourage students to show their work can improve their learning from problem solving and help their students to learn from their mistakes [48-51]. Moreover, the issue of showing work in problem solving has particular relevance for introductory student learning [51]. For example, it has been found that, while grading, many TAs do not require that introductory students show the steps of their solution or explain why they are using certain concepts. On the other hand, they do expect advanced students to show their work and explain their steps [51]. Another study involving TAs' beliefs about the type of example solutions provided to students reveals that many TAs were unlikely to identify features in the problem solutions that the research literature describes as supporting learning goals for students [52]. Because TAs are often responsible for deciding which types of problems to use, both in their current teaching appointments and in possible future roles as faculty members, their beliefs about the pros and cons of posing an introductory physics problem in 
different ways and in different instructional contexts can affect how those types of problems are ultimately used. Thus, identifying the views of the TAs about the way in which a problem is posed can inform TA professional development programs.

\section{F. Focus of our research}

In the study presented here, we describe the findings of an investigation focused on TAs' views about the pros and cons of two introductory problems that are broken into parts and involve the same physics scenario. We focus specifically on the views of physics graduate student TAs about posing problems in two types of broken-into-parts format (problem posed with subproblems provided). One of these problem types does not involve explicit calculation, while the other does. In particular, TAs in a professional development course were asked to reflect upon five problem types for the same introductory mechanics problem scenario in which two of the five problem types were broken-into-parts problems. Other problem types with which they were asked to compare the broken-into-parts problem type in various instructional situations to meet different instructional goals included a traditional textbook problem not broken into parts, a contextrich problem, and multiple-choice problem types. Although the TAs were asked to rank these problem types, in general assuming well-validated problems of each problem type on various physics topics were available, for concreteness, they were presented with an example of each problem type in one context. Here we summarize the TAs' views about two types of broken-into-parts problems and investigate the following research questions: (i) How challenging and instructionally beneficial do TAs perceive the broken-into-parts physics problems to be? (ii) If TAs had complete control of an introductory physics course, how likely would they be to use broken-into-parts problems? (iii) Why do TAs perceive the broken-into-parts problems the way they do?

\section{METHODOLOGY}

\section{A. Participants}

A total of 97 TAs from a typical large research university participated in this study during 4 different years. Participants were physics graduate students who had teaching responsibilities (introductory recitation or lab instruction, and a majority were also assigned to help students in a physics tutoring center) and were concurrently enrolled in a mandatory TA professional development course that met once per week for $2 \mathrm{~h}$ for an entire semester. The TAs were expected to do approximately $1 \mathrm{~h}$ of homework each week pertaining to the professional development course, in which various activities took place throughout the semester. Initial activities related to the course focused on some general issues related to physics teaching and learning, e.g., discussion of some physics education research papers on common student difficulties in introductory physics. The discussion of grading practices occurred near the beginning of the semester, followed by discussions of pedagogy, including the use of tutorials and clicker questions as learning tools and the importance of integrating conceptual and quantitative learning. Next, discussions turned to how different problem types (e.g., multiple-choice problems, context rich problems, problems that are broken into subproblems, and traditional textbook style problems) can help students learn physics and can be useful in different instructional setttings to meet different instructional goals. Before the activities, TAs were involved in evaluating the effectiveness of multiple-choice questions on several standardized conceptual physics surveys, and predicting which choices students might select and why. This activity also gave TAs the opportunity to reflect on the design of conceptual multiple-choice questions, and anticipate challenges their students might encounter. The TAs were also given a physics problem and asked to present the solution to the TA professional development class as they would in their recitations. These presentations were video recorded so that they could reflect on their teaching and also receive feedback from other TAs and the instructor. Thus the problem-type activity was one of a number of activities all aimed at improving the professional development of the TAs and focused on investigating their views about physics teaching and learning.

\section{B. Data collection tools and artifacts}

The data collection tools consisted of instructions and five example introductory physics problem types that had been developed previously to illustrate each problem type [47]. The example problem types were designed for an introductory physics problem scenario in mechanics and served as a guiding example to illustrate what a particular problem type could look like for a given scenario for the activities. They included two different versions of a problem that was broken into subparts (one was framed in a more conceptual manner than the other), a multiple-choice problem, a context-rich problem, and a traditional textbook version of the problem. Here we focus on the two brokeninto-parts problem types for which the example problems given for reference are problem A and problem D in Fig. 1. We note that it was made clear to the TAs several times that these were merely single concrete examples of broken-intoparts problem types for illustration purposes and that they should, in general, reflect upon the instructional benefits of well-designed broken-into-parts problems.

Based upon our research questions, the TAs were asked to answer questions about these problem types on a worksheet in which they were directed to list pros and cons for each problem type. Specifically, in the instructions, TAs were asked to list at least one pro and one con for each problem type based upon the features each of the five problem examples contained. Data were collected over four different years. In the most recent year's worksheet, TAs 


\section{Problem A}

A $1.8 \mathrm{~kg}$ mass is attached to a frictionless pivot point and is moving in a circle at the end of a $65 \mathrm{~cm}$ string. The string breaks when the mass is moving directly upward and the mass rises to a

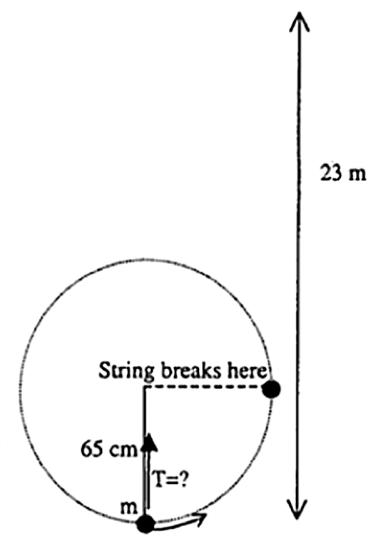

B) What velocity, $v_{0}$, must the stone have when it is at its lowest point in order to have a velocity $v_{1}$ when released?

C) What force will you have to exert on the string at its lowest point in order for the stone to have a velocity $\mathrm{v}_{\mathrm{o}}$ ?

\section{Problem D}

You are whirling a stone tied to the end of a string around in a vertical circle of radius $\mathrm{R}$. You wish to whirl the stone fast enough so that when it is released at the point where the stone is moving directly upward it will rise to a maximum height, $\mathrm{H}$, above the lowest point in the circle. In order to do this, what force will you have to exert on the string when the stone passes through its lowest point one-quarter turn before release? Assume that by the time that you have gotten the stone going and it makes its final turn around the circle, you are holding the end of the string at a fixed position. Assume also that air resistance can be neglected.

A) For each point labeled in the diagram, circle the symbol(s) that describe how the speed of the stone is changing.

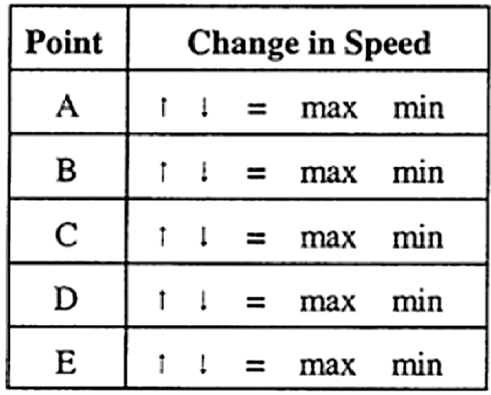

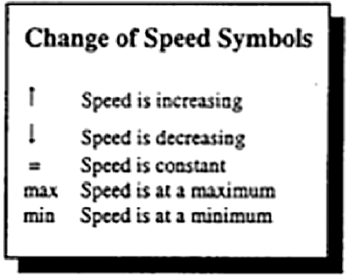

B) At each point on the diagram, draw and label a vector representing the acceleration of the stone.

C) At each point, draw and label vectors to represent all of the forces acting on the stone.

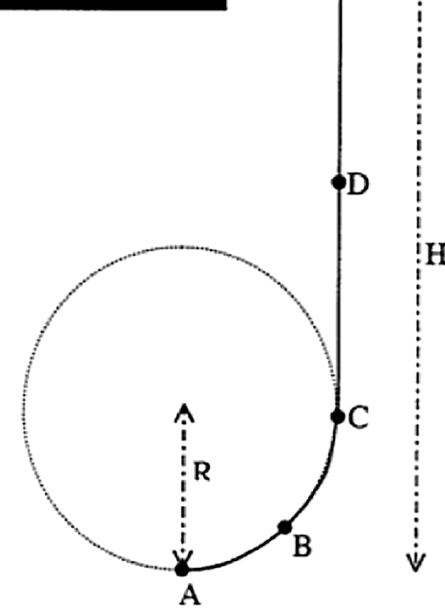

FIG. 1. The two broken-into-parts example problems given to the TAs to illustrate each broken-into-parts problem type. 
were also asked what they would change about the example problems. In addition they were asked to rank the problem types on their instructional benefit (i.e., how instructionally beneficial the TAs judged each problem type to be), and to rank the problem types in terms of the level of challenge (i.e., how difficult the TAs judged each problem type to be for students). They were also asked how much they liked the problem types, and the likelihood that they would use the problem type if they had complete control of the choice of the problem types to use. For example, a TA who ranked a problem 1 for challenging judged this problem to be the least challenging for students; a 5 for challenging indicates that the TA perceived it to be the most challenging among the five problem types. The rankings allowed us to investigate research questions 1 and 2. Furthermore, throughout all four years, TAs were asked to list pros and cons of the problem types. These pros and cons were useful for investigating why TAs ranked the problem types the way they did (research question 3).

\section{Data collection in the TA professional development course and later in individual interviews}

TAs were given the example problem types and worksheets in the professional development course in the middle of the semester, when they had some teaching experience, in order to elicit their written responses about different problem types. They were asked to answer worksheet questions under the assumption that they had complete control over the introductory physics class, including control over problem types chosen for various purposes. They were also told that the actual examples of the problem types were examples and their responses should be about well-designed problems of each type in general. The worksheet was completed as part of a homework assignment. In addition to the written data, qualitative data were collected via interviews with individual TAs. In particular, 12 participants who had completed the TA professional development course earlier volunteered to be interviewed in a one-on-one setting using a semistructured think-aloud protocol about their views on all problem types. The semistructured think-aloud protocol was chosen in order to gather TAs' ideas about the problem types without explicitly steering their opinions with leading questions but being able to probe their responses more in depth than the written responses. These interviews took place at least one semester after the initial activity described here in the TA professional development course and were audio recorded. Since TAs who answered the written questions may have become focused on example problems given to illustrate each problem type, TAs who participated in the interviews were asked questions both about the example problem type and about the problem types in general similar to the broader in-class discussion about the instructional benefits and pros and cons of well-designed broken-into-parts problems. Thus, these interviews served to more deeply probe a subset of TAs' reasoning behind their written responses and to explore such issues as the use of welldesigned broken-into-parts problem types vs other problem types in general. TAs who were interviewed were first asked to complete the same ranking activity as was done in written data collection. After that, the TAs were asked a number of follow-up questions in a semistructured way. Clarification and elaboration was asked of the TAs as deemed useful. Below are the additional interview questions that served as the basis for the semistructured interviews:

1. What about each problem did you like the most? The least? Why?

2. What problem feature(s) of each type of problem do you find most instructionally beneficial? Why?

3. What problem feature(s) of each type of problem do you find the least instructionally beneficial? Why?

4. How often do you think you would use each problem type in homework, quizzes, and exams, if you had complete control of the class? Very often, often, sometimes, rarely or never? Why?

5. How difficult should problems be to be beneficial to student learning in different instructional contexts? Why?

6. Do you feel that introductory students can handle difficult problems? Why or why not? When should students be expected to handle difficult problems?

\section{Coding TA responses}

Two of the researchers met weekly to identify appropriate coding categories for pros and cons; agreements on these were reached through discussion. The researchers used open coding of the data from the individual homework assigned in the middle of the semester regarding the TAs' views of the problem types. The categories coded were arrived at over several weeks based on emergent themes. Some categories were merged if they were found to be sufficiently similar. The interrater reliability was examined for the coding of the pros and cons in the year 3 dataset, and the average Cohen's kappa [53] was calculated to be $\kappa=0.982$. The most common pros and cons for the broken-into-parts problem type, along with their definitions and examples from TA worksheets are included in Table I.

\section{RESULTS}

\section{A. Broken-into-parts problem types ranked high for "like," "use," and "instructional benefit," but low for "challenge"}

Figure 2 summarizes the ranking for all problem types in all four categories in order to compare the TA views of the broken-into-parts problem type with the other types of problems since the rankings are best understood in the context of the other problem types that the TAs were also asked to rank. The average rankings for instructional benefit, indicate that the broken-into-parts problem type 
TABLE I. The most commonly listed pros and cons of the broken-into-parts problem type and the percentages of TAs who listed them. Some TAs listed more than one of the following or other pros and cons not listed here.

\begin{tabular}{lccc}
\hline \hline Code & Definition & Examples & $\begin{array}{c}\text { Percentage } \\
\text { of TAs }\end{array}$ \\
\hline (Pro) guide & $\begin{array}{c}\text { Walks students through step by step; } \\
\text { helps students solve harder problems } \\
\text { Provides too much support or makes } \\
\text { the problem too easy }\end{array}$ & $\begin{array}{c}\text { "parts make the problem more guided" } \\
\text { (Con) help } \\
\text { thent does not have to do }\end{array}$ & 80 \\
\hline \hline
\end{tabular}

is seen by TAs as the most instructionally beneficial of all example problem types. Likewise, the average rankings for like and use indicate a similar sentiment, with both of the broken-into-parts problem examples being ranked the highest of all example problem types. However, the average rankings for challenge reveal that the broken-into-parts problem type is ranked as the least challenging by TAs.

Thus, Fig. 2 shows that the broken-into-parts problem types were consistently ranked as the easiest, and the context-rich problem type was ranked as the most challenging. The multiple-choice and standard "textbook" style problem types were ranked in between these two extremes. The average rankings for like, use, and instructional benefit indicate the opposite, with the context-rich problem type being ranked the lowest of all problem types and the two broken into parts problem types (quantitative and qualitative) ranked the two highest of all problem types, with the multiple-choice and textbook problem types ranked in between.

The two broken-into-parts problem rankings were averaged and a Friedman test was performed to investigate whether there was a statistically significant difference for each category between any of the problem types [54].
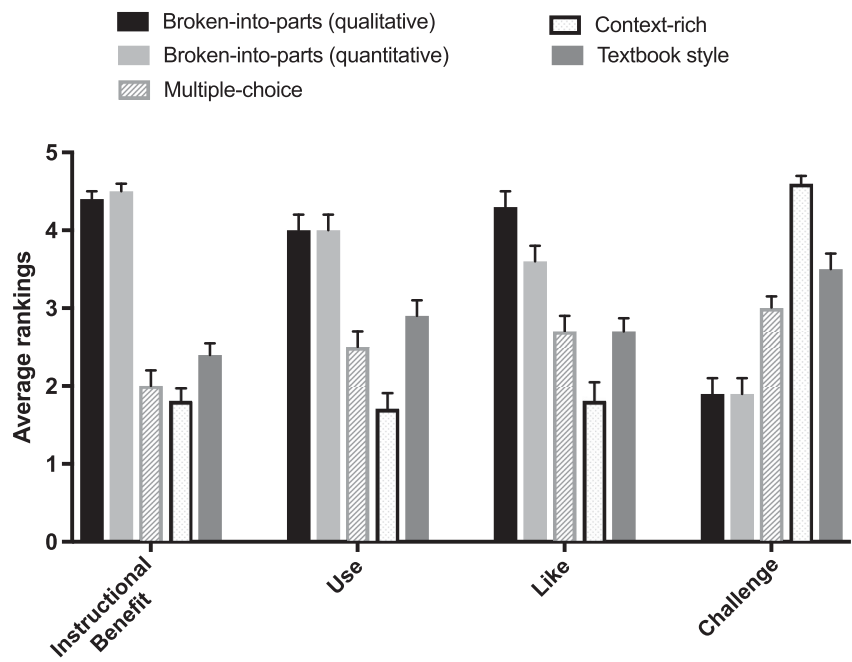

FIG. 2. Average rankings of the two broken-into-parts problem types are higher than the other types of problems for like, use, and instructional benefit, and lower than the other types of problems for challenge.
We found statistically significant differences at $p<$ 0.00001 in all four categories (instructional benefit, use, like, challenge). Therefore, separate tests were conducted to determine statistical significance of the differences between the average broken-into-parts rankings and rankings on each of the other problem types and the $p$ values were adjusted to account for type 1 errors when performing multiple comparisons using the Benjamini and Hochberg procedure [55]. All adjusted $p$ values were statistically significant, with $p \leq$ 0.0.00815 in all cases suggesting that compared to all other problem types, the average broken-into-parts problem type was ranked statistically significantly higher in the categories of instructional benefit, use, and like, and it was ranked statistically significantly lower in the category of challenge.

Since written responses may have been impacted by the example problem types given to the TAs to illustrate different problem types (even though TAs were explicitly asked to think not only about the specific example problems that they were given to illustrate a problem type, but also to think about well-designed problems of each type in general), we asked all interviewed TAs to also rank all problem types. Interviewed TAs responded in a similar manner to those who gave written responses for all of the preceding categories (i.e., instructional benefit, challenge, like, and use) even when explicitly reminded to think more generally about well-designed problems of each type. In other words, rather than the above rankings holding true only for the specific example problems given, interviewed TAs responded similarly when they ranked the different types of problems in a one-on-one interview situation. During class discussion and interviews, some TAs noted that even before the TA professional development class, they have felt that broken-into-parts problems are the best for introductory students, e.g., because those are the types of problems introductory students can be expected to do without becoming stressed and they are clear for students.

\section{B. TAs reported a wide usage of broken-into-parts problem types and preferred to use these problem types over other more challenging problem types}

As seen in Fig. 2, the TAs ranked the broken-into-parts problem types highly for use. This ranking is the highest ranking of all problem types the TAs considered. Both of the broken-into-parts problem types received an average 


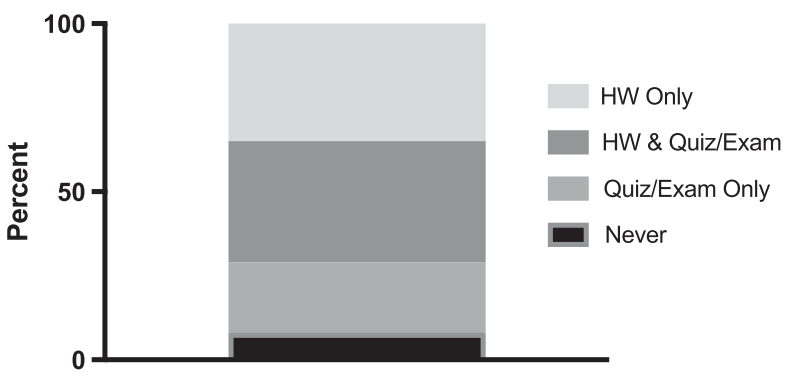

FIG. 3. TAs' reported usage of the broken-into-parts problem type. These are the reported usages as averaged over both examples of the broken-into-parts problem type since there was no significant difference between the two broken-into-parts problem types. Light gray indicates the TAs who would only use this type of problem in homework. Dark gray indicates those who would use it in homework, quiz, or exam. Medium gray indicates those who would use it in quize or exam only. Black indicates those who would never use it for any purpose.

ranking of 4 out of 5, indicating that TAs were far more likely to use this problem type compared to other problem types, the next highest ranking for which was only a 2.9 out of 5 in the category of use.

Figure 3 summarizes the TAs' stated use of the brokeninto-parts problem types (taken together), and shows that TAs reported that they would readily use these types of problems for homeworks, quizzes, and exams. However, written responses and interview data hint at possibly excessive valuing and use of this type of problem, indicating a potential overreliance on broken-into-parts problems. For example, in an interview, one TA stated: "I always prefer subquestions" whether it is for homework, quizzes, or exams. Further discussion with the TA suggests that he would almost exclusively use broken-into-parts problems and was not likely to use other problem types, which may provide less support and create more of a challenge for the introductory physics students especially in exams. Similarly, regarding the broken-into-parts problem type, another interviewed TA stated: "I will use it everywhere." The idea of almost-exclusive preference for using this type of problem, particularly for exams, was conveyed by many other TAs during the interviews, as well as in written responses. Below, we discuss reasons behind the rankings and stated uses, based upon interview data and the written responses in the columns of the worksheet which asked for explanations and/or reasons for their responses.

\section{TAs viewed the pro of guiding students as outweighing the con of providing too much help}

The written pros and cons and explanations as well as the interview data were analyzed regarding the brokeninto-parts problem type for possible reasons for why the TAs ranked the broken-into-parts problem type the way they did. Table I shows the most common pros and cons mentioned by TAs in written responses.
The most common pro stated for the broken-into-parts problem type was "guide," which was mentioned by $80 \%$ of TAs. "Guide" was the category used for TAs' responses which included what they judged to be an opportunity to guide the students in the problem solving process. Some examples include: "Guides students to understand how to solve the problem," and "Leads the student to solve the problem step-by-step."

The prevalence of the pro guide could explain why TAs ranked the broken-into-parts problem type highly in terms of instructional benefit. One TA stated: "Very instructional ... break-down helps students solve problem." The TAs with this type of response appreciated the support provided in these types of problems because it was perceived as a way to guide students in solving the problem. Moreover, of all the TAs who mentioned the pro guide for problems A and D, all but one TA ranked problems A and D as the two highest out of all the problem types they were given, in terms of their instructional benefit.

One TA described his reason for judging the broken-intoparts problem type as being highly instructionally beneficial by stating that he prefers to give "questions that the student can just jump in and start immediately. And later, they look at their results from one part and can get some inspiration for the next part." This TA further explained how a broken-into-parts problem can make the problemsolving process more manageable for introductory physics students by providing them with support to get from one step to the next through the solution. In his view, many introductory physics students would otherwise not be able to solve the problems.

By contrast, TAs did not list very many cons. Even though they were specifically asked to list at least one pro and one con, many TAs failed to list any cons for the broken-into-parts problem type completely. Table I shows that the only commonly stated con for the broken-into-parts problems A and D was "help," and that this con was mentioned by only $37 \%$ of TAs. The category help contained TA responses in which the TA expressed concerns that breaking a problem into subproblems for students may make this type of problem too easy (for example: "not difficult enough" or "helping the student too much"). Thus, the con help suggests that some TAs had reservations about the broken-into-parts problem type potentially providing too much help to students, but this con is mentioned by only about one-third of TAs in written response (even though the TAs were asked to mention at least one con).

Even in interviews, any con for broken-into-parts problems was rarely mentioned. Moreover, interviews suggest that the possibility that broken-into-parts problems may not be difficult enough was a minor concern to most interviewed TAs and they were consistent in noting that it would not deter them from giving priority to this type of problem over other types on homework, quizzes and 
exams. For example, one TA reported in an interview that "D might be too easy." However, he went on to say that, despite that "... actually, [it] is good in the sense that it's like a good way of testing the students' conceptual understanding ...." He went on to discuss the merits of breaking a problem into parts for a student, stating: "It kind of leads you through all the steps ... so [for an introductory class] I would..give them this," indicating that he appreciated the guidance the problem provides. He further suggested that his concern about these problems being "too easy" was outweighed by the value he saw in them for the introductory physics students. Another TA initially expressed some reservation by stating that the broken-into-parts problems "may be setting up a problem a little too much ..." but then also ranked the broken-into-parts problem type the highest in the category like and cited the guidance offered by this problem type as a major pro. He further explained that these types of problems were appealing to him because of the "... multiple parts to guide students" and that he would certainly give priority to using such problems in homework, quizzes, and exams in introductory physics. Further discussion suggests that the TA's overall impression of the broken-into-parts problems was very positive, and his concern about helping students too much appears to be overshadowed by the positive aspects he perceived in this type of problem.

Other TAs expressed similar sentiments about the pros being more compelling than the cons of broken-into-parts problems (if they mentioned a con at all, which was only roughly one-third of the TAs). Both written and interview data suggest that the con help may not be viewed as a major drawback to TAs. Indeed, TAs were often reluctant to report downsides to a broken-into-parts problem type, sometimes using superlative language to describe this type of problem. For example, several TAs went as far as to use the word "perfect" in describing broken-into-parts problems for homework, quizzes, and exams in introductory physics, apparently not detecting any drawbacks to this type of problem. Moreover, although TAs mentioned the asset of a broken-into-parts problem type guiding the introductory physics students in solving the problem at hand, the nature of the responses did not usually indicate the idea that such problems could be used to provide guidance to students to solve future problems that are not broken into parts. Furthermore, TAs rarely mentioned in interviews or written responses that the scaffolding support provided by these types of problems should gradually be removed to help students develop self-reliance in problem solving.

\section{TAs had different expectations of introductory and advanced students}

Despite the low ranking for challenging, the majority of TAs reported a preference for broken-into-parts problems, ranking them highly in the category like. Our follow-up interviews indicated that many TAs felt that introductory physics students should not be given problems that were overly challenging (e.g., a problem scenario which is not broken into parts) partly because they did not expect introductory students to be able to break up the problem into subproblems on their own. On the other hand, when TAs were asked during interviews whether they would use broken-into-parts problems for advanced students if they were teaching an advanced physics course, many reported that such problems would be "too easy" for advanced students even in the context of their advanced physics problem solving, and therefore such broken-into-parts problems should not be used or rarely be used in advanced courses. It is important to note that, in the interviews, TAs were specifically asked whether appropriate advanced topic broken-into-parts problems should be used for advanced students. Thus, it was not the introductory physics topic that TAs felt was too easy for advanced students. Rather, it was the support provided by the problem being decomposed into subproblems that TAs felt was not appropriate for advanced physics students, even if the problem dealt with an advanced-level topic. Thus, there was a discrepancy between the TAs' expectations of introductory students with regard to introductory-level physics problem solving compared to advanced students with regard to advancedlevel physics problem solving.

Exhibiting a relatively low expectation level for introductory physics students, one TA said, "My gut feeling is that you don't want to knock the [introductory] students out with a tough problem." This TA further suggested that challenging problems should be avoided in introductory physics in general, and that he felt that introductory students are unlikely to be able to handle difficult problems. Another TA who ranked the broken-into-parts problem type the highest in the category like explained that "This would be, for me as an intro student, the ideal problem, because I have to use my skills to be able to translate what I'm being asked into the mathematical formulas, but at the same time I have enough guidance..." This TA felt that broken-intoparts problems provide the appropriate level of difficulty for introductory physics students. Likewise, in explaining his preference for a broken-into-parts problem type, another TA stated: "Trying to unpack a problem into different parts is half the battle sometimes with solving these problems. When it's done for you that's helpful, and it's really important on a test too..." This TA felt that making the problem less difficult for introductory students was important even in a test situation. He believed that the challenge of "unpacking" the problem should be done for the introductory student, in homework, quizzes, and exams, rather than expecting that the students do this unpacking themselves. He expressed lack of confidence that most introductory students could accomplish this task on their own and if we wanted to see some work for each problem posed, breaking them into parts was a good way to accomplish it. In a similar way, another TA who ranked 
the problem high for like and low for its level of challenge stated: "I like that the problem is broken down into small questions that help you solve the original problem. For introductory classes, [even] this may be a bit difficult." This TA expressed concern that while these types of problems are easier than problems which are not broken into parts, even this level of challenge is potentially very difficult for most introductory students in any context, indicating a relatively low expectation level regarding the types of problems introductory physics students should be expected to handle in homework, quizzes, and exams.

Most TAs did not identify when introductory physics students should be expected to learn how to break physics problems into subproblems themselves. They did not report that problems with less support would be important to use in order to help introductory students gain more independence in problem solving by developing skill in decomposing a problem into subproblems on their own. One TA who reported that he really likes and would widely use a brokeninto-parts problem explained, "Students can be instructed while doing the problem and, while it doesn't have them connect the steps themselves, that probably isn't the point." Thus, while this TA momentarily considered the fact that this type of problem did not require students to connect the steps of the problem-solving process themselves, further discussion suggested that he did not regard "connecting the step" as a crucial component of the types of problems introductory students should be expected to solve. He also did not indicate during the discussions that the independent practice of connecting the step and learning to break a problem into subproblems are critical steps towards developing expertlike problem-solving skills.

Most interviewed TAs did not find a broken-into-parts problem type to be appropriate for advanced students (unlike introductory students). It appears from interviews as though the TAs believed that advanced physics students should be able to decompose a problem into subproblems on their own. However, most interviewed TAs did not mention that introductory students should independently practice the skill of decomposing introductory problems into subproblems in order to develop expertise in problem solving. For example, one TA explained the discrepancy between expectations for introductory vs advanced students as follows: "very much, because the steps lead the students to solve the problem, but this knowledge is basic and too easy for an advanced level student." This TA expressed an expectation that advanced students should be able to break an introductory physics problem down into subproblems on their own, but that introductory students should not be expected to do so. This TA mentioned advanced students' ability to break and introductory physics problem down into subproblems, but some TAs also mentioned that advanced students should be able to break advanced physics problems down into subproblems on their own. Most interviewed TAs with this type of response did not explicate that some problems with less support for introductory students would be a way to cultivate expertlike problem-solving skills and had relatively low expectations of the types of problems introductory students could be expected to solve.

\section{E. TAs' preference for broken-into-parts problems may be influenced by introductory students' preferences}

Both the broken-into-parts problem types were strongly preferred by the TAs compared with other problem types, as evidenced by the average rankings in the category like. Both written and interview data suggest that one of the reasons for this preference could be the TAs' interest in what they believed introductory students will like. In particular, introductory students might prefer problems that are easier for them to solve, and this preference appeared to be on the minds of the TAs. For example, one interviewed TA who had ranked the broken-into-parts problems as the highest for like expressed that, "Breaking it into parts is reducing the workload for the students and I think they're going to appreciate that." This TA indicated that lightening the workload for introductory students entered into his thinking, suggesting that pleasing students in this way may be at least part of why he valued these types of problems for homework, quizzes, and exams. Another TA who ranked the broken-into-parts problems highly in the category like and noted that he likes these types of problems for homework, quizzes, and exams stated in the interview: "I think students would like this one [problem A] most." Again the idea of what students might prefer appeared to contribute to the TA's preference for these types of problems. Some of them were concerned that if the problems were not broken into parts, introductory students may get frustrated with the problems and with the TA. Most of the other interviewed TAs had similar concerns about introductory students' preferences. One interviewed TA tried to explain why he liked broken-into-parts problem types in all situations by stating that, for such problems, "If the basics are clear, they [introductory physics students] will sail through." He emphasized that making sure that introductory physics students sail through the problems was his main goal. While this TA acknowledged that this problem type is easier than problems which are not broken into parts, he thought they were "nice" problems for use in homework, quizzes, and exams so that introductory students do not have to struggle too much and he ranked it highly for like and use. It appears that the ease with which students could solve such problems was a factor in his preference for a broken-into-parts problem. Other interviewed TAs appeared to convey similar sentiments regarding making problems easier for the introductory physics students so that they do not get frustrated and liked the TAs by way of breaking problems into parts before posing them. 


\section{DISCUSSION AND SUMMARY}

This study provides a snapshot of TAs' perceptions of broken-into-parts problem types vis-à-vis other problem types half way through a TA professional development course (written data) and at the end of the course (interview data) at a large research university. We note that TAs in the study belonged to different cohorts, and their responses were consistent across time. We find that most TAs ranked the broken-into-parts problem type highly and stated that they would use this problem type often on homework, quizzes, and exams because breaking the problem into subproblems before posing it is necessary to facilitate the problem-solving process for introductory students. Some TAs did not write any con for the broken-into-parts problem type even when explicitly asked. Some noted that they will almost exclusively use these types of problems particularly in exams. Discussion during interviews suggests that TAs may overuse broken-into-parts problems partly due to their preference to guide introductory physics students through the problem-solving process, their relatively low expectation level for introductory students, and their consideration of introductory students' preferences (such as reducing their students' stress while solving physics problems). However, TAs did not indicate that introductory students should also be given opportunity to practice more independent problem solving via problems in which the scaffolding support is removed after the modeling and coaching part of the cognitive apprenticeship process in order to develop self-reliance. In the cognitive apprenticeship model, appropriate coaching and scaffolding support can help develop expertise and provide guidance to students to eventually gain independence in solving complex physics problems [18]. This type of long-term goal of developing expertise of introductory students via more complex physics problems that were not broken into parts was not typically mentioned or implied by TAs' responses in written or interview data. Instead, the use of a brokeninto-parts problem type was regarded by TAs as beneficial and necessary for helping guide students in solving the problem at hand in most contexts and this pro (along with reducing stress for students) appears to be a major reason for why the TAs were likely to frequently use broken-intoparts problems in homework, quiz, and exam situations.

These findings partly agree with a similar study involving physics instructor's views of various problem types, in that, like instructors, TAs reported extensive use of brokeninto-parts problems despite any reservations they might have about them [47]. However, the TAs in this study appear to have an even stronger preference for the brokeninto-parts problem type than did the faculty members in that fewer TAs expressed a concern that such problems may provide too much help to students (even when explicitly asked to state at least one con of a broken-into-parts problem type) compared with the number of faculty members who expressed similar concerns. While nearly half of faculty members identified independent problem solving without guidance as an important goal in teaching problem solving [47], few TAs mentioned that using problems which do not provide introductory students with guiding support was important because they can help introductory physics students develop self-reliance in problem solving. Additionally, interviews and written data suggest that, even among those TAs who had a concern about a broken-into-parts problem potentially providing too much help, this concern was not strong and did not outweigh the benefit of guiding a student through a problem by breaking it into parts in homework, quizzes, and exams. Moreover, while both TAs and faculty members reported copious use of broken-into-parts problems with their introductory students whether or not they had concerns about such problems, most TAs overlooked the need to challenge introductory students by offering them opportunities to solve problems which do not have the steps already broken down for them so that they can develop expertise and self-reliance in problem solving.

Interviews also suggest that TAs expected advanced students to be capable of decomposing an advanced problem on their own. Yet, if introductory students do not practice breaking down an introductory physics problem into sub-problems on their own, they may not be able to develop this skill on their own and become effective problem solvers. TAs' perspectives appeared to be missing the crucial bridge between highly supported problem solving and independent problem solving for introductory students. Without this step, introductory students can be severely hindered in their development of expertise in problem solving, reasoning, and metacognitive skills. This missing puzzle piece is similar to the discrepancy in TAs' grading practices in a prior investigation in that TAs did not demand that introductory students explicate the steps in their solutions (and would not penalize them for neglecting to show work), but expected advanced students to do so in advanced physics and would penalize them if they did not do it [51]. In particular, in a previous study related to TAs' views about grading practices, many TAs felt that advanced students should be required to show steps and reasoning in their advanced physics problem solutions but claimed that introductory students need not show steps or provide conceptual reasoning in their introductory physics solutions and should not be penalized for omitting such steps [51].

This study suggests that TAs had a relatively low expectation of introductory students' problem solving skills and had not reflected on ways in which introductory students can be provided scaffolding support and guidance to cultivate independent problem solving skills. Such skills (e.g., breaking a problem into subproblems on one's own) are unlikely to develop spontaneously and must be explicitly cultivated by incorporating them into instructional design and having high expectations of introductory students while helping them develop self-reliance in 
physics problem solving. TAs' preference for continually providing problems for introductory students which are broken into parts represents an important oversight in the steps required for introductory students to learn independent expertlike problem solving. In particular, introductory students must be given opportunities to practice bridging the gap between solving problems that are broken into parts and solving problems with less built-in support, a point that most TAs appear to have missed.

\section{IMPLICATIONS FOR PROFESSIONAL DEVELOPMENT OF TEACHING ASSISTANTS}

These findings that provide a snapshot of graduate TA views at a large research university (half way through their first semester TA professional development course and at the end) can be valuable to inform the design of professional development of TAs at similar universities with similar professional development programs in order to improve introductory physics student learning and ensure expertise development. Leaders of TA professional development programs at similar universities can incorporate the findings of this study to help TAs create appropriate teaching and learning goals for introductory physics students that support student growth and learning and reflect on instructional approaches that support the goals. For example, our findings indicate that TAs had relatively low expectations for what introductory students can accomplish while solving physics problems. Our findings suggest that most TAs may not have thought about the goal of helping introductory students become independent problem solvers and the important role they can play by setting high expectations (and communicating this to students) and providing them with scaffolding support with a focus on reducing the support to help them develop self-reliance. To help TAs readjust their expectations of introductory students, in a professional development course or program, TAs can be asked to reflect on and clarify their learning goals for introductory students individually and then in small groups with facilitation from the course instructor. They can also examine how different problem types can support (or hinder) achievement of different learning goals in different instructional contexts. In particular, TAs may be given opportunities to discuss how broken-into-parts problems support the goal of helping introductory students learn, e.g., how to decompose problems into subproblems in the modeling and coaching phases of the cognitive apprenticeship model to help students develop expertise. In addition, TAs can be given opportunity to reflect upon and discuss as a group how other problem types which do not decompose the problem into sub-problems may support the goal of helping introductory students develop selfreliance in problem solving. They can also reflect on their differing expectations of advanced physics students with regard to advanced physics problem solving and introductory students with regard to introductory physics problem solving and why having a lower expectation of what introductory students can learn and be able to do while solving an introductory problem can be detrimental to their overall learning and growth in introductory courses. In the professional development programs, TAs can be asked to contemplate and discuss how the use of different introductory problem types in different instructional contexts can accomplish various instructional goals and can help introductory students progress toward expertlike problem solving approaches. In this way, TAs may begin to appreciate that, while broken-into-parts problems are an important stepping stone in the development of expertlike problem solving, other problem types that do not provide scaffolding support are also critical in the development of self-reliance in problem solving.

\section{LIMITATIONS AND FUTURE DIRECTIONS}

While this investigation involving graduate TAs at a large research university in their first semester TA professional development course (in the middle and at the end of the course) sheds light on the potential that TAs may over rely on the use of broken-into-parts problems that may be detrimental to helping introductory students develop expertise in physics problem solving, below, we note the limitations of the study that should be kept in mind and future directions that could add to these findings.

First, the findings and implications for professional development of TAs are most relevant only to large institutions that have an established TA professional development course similar to ours. In particular, our findings may not be representative of TA responses that would be generated from institutions which are not similar to ours and in which similar TA professional development is not there. It would be valuable for researchers to carry out future studies at other types of institutions and compare them to our findings to determine how generalizable the results are across different institutions.

Second, our findings provide a snapshot of graduate TAs' views half way through the TA professional development course (written data) and at the end of the course (interview data). Moreover, although some interviewed TAs in our study mentioned on their own that they had similar views about the great potential of the broken-intoparts problems for introductory physics courses even before they started graduate school, and the findings from both written and interview data were consistent with each other for cohorts over several years, it is possible that TA responses would change over a longer period of time. Therefore, future studies would explore how TAs' ideas about various problem types may evolve over time by carrying out interviews with the same TA several times extended over many years.

Third, while the study involved both written and interview data, the interviews were semistructured think-aloud interviews. In particular, the current study highlights the 
fact that in response to written questions and interviews, TAs rarely readily and spontaneously articulated the idea that the scaffolding support provided by these types of problems should gradually be removed to help students develop self-reliance in problem solving. In think-aloud interviews, while we asked students to elaborate and clarify their responses and thought processes to the questions posed to get an in-depth understanding of their responses, these were not dynamic interviews [56], e.g., which explicitly drew attention to the fact that the TAs may be missing the value of helping introductory students develop expertise in physics problem solving by gradually removing the scaffolding support and helping students develop self-reliance. In other words, while the interviews using think-aloud protocol had the advantage of allowing TAs to express their candid opinions without leading questions if they missed something, this protocol limited interviewer's ability to explicitly ask questions regarding aspects of the broken-into-parts and other problem types that TAs may not have mentioned on their own. While there is considerable value in probing what TAs will articulate with minimal prompting beyond followup to what the TAs stated in response to the questions, more explicit followup questions could help give a better sense of TAs ideas about how the question types should be used over time or how, if at all, students could be helped to develop selfreliance in problem solving. Thus, while such questions were not included due to the original design of the study focused on TAs' views with minimal intervention, responses to such questions could help clarify TAs' views further. Therefore, a future direction that would further elucidate the findings presented here would be to conduct dynamic interviews [56] with the TAs. Such interviews would include focused questions that would examine issues that TAs did not explicitly mention on their own in response to the questions they were asked. For example, these interviews could include questions about the ways in which
TAs might consider how, if at all, the use of different types of problems might facilitate or hinder their students' development of self-reliance in problem solving even if the TAs never mentioned it spontaneously.

Moreover, data collection during an ongoing, not-yetcompleted TA course, could potentially have impacted TA responses in certain ways and the relatively low sample size associated with that TA professional development course highlights the challenge of doing research in such environments. Future studies can try to account for these challenging limitations and whether and how they impact TA responses.

Finally, we note that in Ref. [47], individual interviews were carried out with faculty members who had significant experience teaching introductory physics courses independently and the focus of those interviews was on how the different problem types fit in the current teaching practices of a faculty member. Since graduate TAs in their first semester did not have independent teaching experience, all questions were framed in a hypothetical context in which the TAs had full control of teaching the introductory physics course. Thus, the way interview questions were framed for the faculty members was not appropriate for our study with the TAs. In particular, it should be kept in mind that the difference in the way interview questions were framed may have led to the fact that while nearly half of interviewed faculty members identified independent problem solving without guidance as an important goal in teaching problem solving, few TAs mentioned that using problems which do not provide introductory students with guiding support was important because they can help introductory physics students develop self-reliance in problem solving.

\section{ACKNOWLEDGMENTS}

We thank the National Science Foundation for Grant No. PHY-1806691. We thank Xu Qin, Danny Doucette, and Kyle Whitcomb for helpful discussions.
[1] B. Eylon and F. Reif, Effects of knowledge organization on task performance, Cognit. Instr. 1, 5 (1984).

[2] L. Hsu, E. Brewe, T. Foster, and K. Harper, Resource Letter RPS-1: Research in problem solving, Am. J. Phys. 72, 1147 (2004).

[3] J. Mestre, Is transfer ubiquitous or rare: New paradigms for studying transfer, AIP Conf. Proc. 790, 3 (2005).

[4] D. Meltzer, Relation between students' problem solving performance and representational mode, Am. J. Phys. 73, 463 (2005).

[5] M. Scott, T. Stelzer, and G. Gladding, Evaluating multiplechoice exams in large introductory physics courses, Phys. Rev. ST Phys. Educ. Res. 2, 020102 (2006).
[6] K. Harper, Student problem solving behaviors, Phys. Educ. 44, 250 (2006).

[7] E. Yerushalmi, C. Henderson, K. Heller, P. Heller, and V. Kuo, Physics faculty beliefs and values about the teaching and learning of problem solving. I. Mapping the common core, Phys. Rev. ST Phys. Educ. Res. 3, 020109 (2007).

[8] C. Singh, When physical intuition fails, Am. J. Phys. 70, 1103 (2002).

[9] M. Kryjevskaia, M. Stetzer, and P. Heron, Student understanding of wave behavior at a boundary: The relationships among wavelength, propagation speed and frequency, Am. J. Phys. 80, 339 (2012). 
[10] K. K. Mashood and V. Singh, Large-scale studies on the transferability of general problem-solving skills and the pedagogic potential of physics, Phys. Educ. 48, 629 (2013).

[11] Z. Chen and G. Gladding, How to make a good animation: A grounded cognition model of how visual representation design affects the construction of abstract physics knowledge, Phys. Rev. ST Phys. Educ. Res. 10, 010111 (2014).

[12] G. Gladding, B. Gutmann, N. Schroeder, and T. Stelzer, Clinical study of student learning using mastery style versus immediate feedback online activities, Phys. Rev. ST Phys. Educ. Res. 11, 010114 (2015).

[13] N. Schroader, G. Gladding, B. Guttman, and T. Stelzer, Narrated animated solution videos in a mastery setting, Phys. Rev. ST Phys. Educ. Res. 11, 010103 (2015).

[14] H. Simon, Models of Thought (Yale University Press, New Haven, CT, 1979).

[15] C. Singh, Assessing student expertise in introductory physics with isomorphic problems. I. Performance on a nonintuitive problem pair from introductory physics, Phys. Rev. ST Phys. Educ. Res. 4, 010104 (2008).

[16] C. Singh, Assessing student expertise in introductory physics with isomorphic problems. II. Effect of some potential factors on problem solving and transfer, Phys. Rev. ST Phys. Educ. Res. 4, 010105 (2008).

[17] S. Y. Lin and C. Singh, Using isomorphic problems to learn introductory physics, Phys. Rev. ST Phys. Educ. Res. 7, 020104 (2011).

[18] A. Collins, J. S. Brown, and S. E. Newman, Cognitive Apprenticeship: Teaching the craft of reading, writing, and mathematics in Knowing, Learning, and Instruction (Lawrence Erlbaum, Hillsdale, NJ, 1989), pp. 453-494.

[19] A. Schoenfeld, Learning to think mathematically: Problem solving, metacognition, and sense-making in mathematics, in Handbook for Research on Mathematics Teaching and Learning, edited by D. Grouws (MacMillan, New York, 1992), pp. 334-370.

[20] E. A. Silver, Foundation of cognitive theory and research for mathematics problem solving instruction, in Cognitive Science and Mathematics Education, edited by A. H. Schoenfeld (Erlbaum, Hillsdale, NJ, 1987), pp. 33-60.

[21] P. Heller, R. Keith, and S. Anderson, Teaching problemsolving through cooperative grouping. Part 1: Group vs. individual problem solving, Am. J. Phys. 60, 627 (1992).

[22] P. Heller and M. Hollabaugh, Teaching problem-solving through cooperative grouping. Part 2: Designing problems and structuring groups, Am. J. Phys. 60, 637 (1992).

[23] F. Reif, Millikan Lecture 1994: Understanding and teaching important scientific thought processes, Am. J. Phys. 63, 17 (1995).

[24] L. Ding, Verification of causal influences of reasoning skills and epistemology on physics conceptual learning, Phys. Rev. ST Phys. Educ. Res. 10, 023101 (2014).

[25] M. Chi, P. Feltovich, and R. Glaser, Characterization and representation of physics problems by experts and novices, Cogn. Sci. 5, 121 (1981).

[26] J. Sweller, Cognitive load during problem solving, Cogn. Sci. 12, 257 (1988).

[27] P. Black and D. Wiliam, Assessment and classroom learning, Assessment in Education: Principles, Policy, and Practice 5, 7 (1998).
[28] C. Singh, What can we learn from PER: Physics Education Research?, Phys. Teach. 52, 568 (2014).

[29] For example, see http://www.aps.org/programs/education / graduate.

[30] J. Nyquist, R. Abbott, and D. Wulff, The challenge of TA training in the 1990s, in New Directions for Teaching and Learning (Kendall/Hunt publishing Company, Dubuque, IA, 1989), Vol. 39, pp. 7-13.

[31] For example, see http:/www.aps.org/programs/education/ graduate/conf2008/index.cfm.

[32] R. Goertzen, R. Scherr, and A. Elby, Respecting tutorial instructors' beliefs and experiences: A case study of a physics teaching assistant, Phys. Rev. ST Phys. Educ. Res. 6, 020125 (2010).

[33] J. Chini and A. Al-Rawi, Alignment of TAss' beliefs with practice and student perception, AIP Conf. Proc. 1513, 98 (2013).

[34] M. Wilcox, Y. Yang, and J. Chini, Quicker method for assessing influences on teaching assistant buy-in and practices in reformed courses, Phys. Rev. Phys. Educ. Res. 12, 020123 (2016).

[35] E. Marshman, R. Sayer, C. Henderson, E. Yerushalmi, and C. Singh, The challenges of changing teaching assistants grading practices: Requiring students to show evidence of understanding, Can. J. Phys. 96, 420 (2018).

[36] Recruiting and Educating Future Physics Teachers: Case Studies and Effective Practices, edited by C. Sandifer and E. Brewe (American Physical Society, PhysTEC, 2015), ISBN: 978-0-9848110-5-2.

[37] M. Good, E. Marshman, E. Yerushalmi, and C. Singh, Physics teaching assistants' views of different types of introductory problems: Challenge of perceiving the instructional benefits of context-rich and multiple-choice problems, Phys. Rev. Phys. Educ. Res. 14, 020120 (2018).

[38] A. Maries and C. Singh, Improving one aspect of pedagogical content knowledge of teaching assistants using the TUG-K, Phys. Rev. ST Phys. Educ. Res. 9, 020120 (2013).

[39] A. Maries and C. Singh, Performance of graduate students at identifying introductory students' difficulties with kinematics graph, in Proceedings of the Physics Education Research Conference, College Park, MD (2015), p. 171, https://doi.org/10.1119/perc.2014.pr.039.

[40] A. Maries and C. Singh, Teaching assistants performance at identifying common introductory student difficulties in mechanics revealed by the Force Concept Inventory, Phys. Rev. Phys. Educ. Res. 12, 010131 (2016).

[41] N. Karim, A. Maries, and C. Singh, Exploring one aspect of pedagogical content knowledge of teaching assistants using the Conceptual Survey of Electricity and Magnetism, Phys. Rev. Phys. Educ. Res. 14, 010117 (2018).

[42] F. Lawrenz, P. Heller, and R. Keith, Training the teaching assistant: Matching TA strengths and capabilities to meet specific program goals, J. Coll. Sci. Teach. 22, 106 (1992).

[43] C. Singh, Categorization of problems to assess and improve proficiency as teacher and learner, Am. J. Phys. 77, 73 (2009).

[44] C. Singh, Rethinking tools for training teaching assistants, AIP Conf. Proc. 1179, 59 (2009). 
[45] A. Mason and C. Singh, Surveying graduate students attitudes and approaches to problem solving, Phys. Rev. ST Phys. Educ. Res. 6, 020124 (2010).

[46] E. Marshman et al., From instructional goals to grading practices: The case of graduate teaching assistants, arXiv: 1601.08210 .

[47] E. Yerushalmi, K. Heller, P. Heller, and C. Henderson, Instructors' reasons for choosing problem features in a calculus-based introductory physics course, Phys. Rev. ST Phys. Educ. Res. 6, 020108 (2010)

[48] E. Yerushalmi, E. Marshman, A. Maries, C. Henderson, and C. Singh, Grading practices and considerations of graduate students at the beginning of their teaching assignment, in Proceedings of the Physics Education Research Conference, College Park, MD (2015), https://doi.org/ 10.1119/perc.2014.pr.024.

[49] C. Henderson, E. Marshman, A. Maries, E. Yerushalmi, and C. Singh, Instructional goals and grading practices of graduate students after one semester of teaching experience, in Proceedings of the Physics Education Research Conference, College Park, MD (2015), p. 111, https:// doi.org/10.1119/perc.2014.pr.024.

[50] E. Yerushalmi, R. Sayer, E. Marshman, C. Henderson, and C. Singh, Physics graduate teaching assistants' beliefs about a grading rubric: Lessons learned, in Proceedings of the Physics Education Research Conference, Sacramento, CA (2016), https://doi.org/10.1119/perc.2016.pr.097.

[51] E. Marshman, R. Sayer, C. Henderson, and C. Singh, Contrasting grading approaches in introductory physics and quantum mechanics: The case of graduate teaching assistants, Phys. Rev. Phys. Educ. Res. 13, 010120 (2017).

[52] S. Y. Lin, C. Henderson, W. Mamudi, C. Singh, and E. Yerashalmi, Teaching assistants' beliefs regarding example solutions in introductory physics, Phys. Rev. ST Phys. Educ. Res. 9, 010120 (2013).

[53] J. Cohen, A coefficient of agreement for nominal scales, Educ. Psychol. Meas. 20, 37 (1960).

[54] M. Friedman, The use of ranks to avoid the assumption of normality implicit in the analysis of variance, J. Am. Stat. Assoc. 32, 675 (1937).

[55] Y. Benjamini and Y. Hochberg, Controlling the false discovery rate: A practical and powerful approach to multiple testing, J. R. Stat. Soc. Ser. B 57, 289 (1995).

[56] N. Rebello, D. Zollman, A. Allbaugh, P. Engelhardt, K. Gray, Z. Hrepic, and S. F. Itza-Ortiz, Dynamic Transfer: A Perspective from Physics Education Research, in Transfer of Learning: Research and Perspectives, edited by J. Mestre (Information Age Publishing, Greenwich, 2004). 